\title{
Descripción de la hembra de Andinosaura kiziarani (Sauria: Gymnophthalmidae) topotípica del sector de Girón, Ecuador
}

\author{
José Manuel Falcón, Juan C. Sánchez-Nivicela ${ }^{1,2,3 *}$
}

\begin{abstract}
1 Universidad Nacional de Colombia, Facultad de Ciencias, Bogotá D.C., Colombia; Grupo de Investigación Evolución y Ecología de Fauna Neotropical.E-mail: juan.sanchezn13@gmail.com. ORCID: 0000-0001-7971-1216

2 Universidad San Francisco de Quito USFQ, Colegio de Ciencias Biológicas y Ambientales COCIBA, Laboratorio de Zoología Terrestre y Museo de Zoología, Campus Cumbayá, Quito 170901, Ecuador ${ }^{3}$ Instituto Nacional de Biodiversidad, Museo Ecuatoriano de Ciencias Naturales, División de Herpetología, Rumipamba 341 y Av. de los Shyris. Casilla 17-07-8976, Quito, Ecuador *Autor para correspondencia / Corrersponding author: juan.sanchezn13@gmail.com
\end{abstract}

\section{Description of the topotypic Andinosaura kiziarani (Sauria: Gymnophthalmidae) female from the Girón sector, Ecuador}

\begin{abstract}
We describe the female of Andinosaura kiziriani from a topotypical locality, in the "El Chorro" sector, southwest of the Azuay province, Ecuador. The female was active on litter and rocks, nearby the El Chorro waterfall. The individual differs from males by presenting 31 transverse rows of dorsal scales, absence of femoral pores, a snout-vent length of $66.1 \mathrm{~mm}$ and a dorsal pattern of dark brown coloration with white dorsolateral bands, and venter dark brown with white stripes arranged longitudinally. Our results document the sexual dimorphism in this species and expands the knowledge of its morphological and ecological characteristics.
\end{abstract}

Keywords: Microteid lizard, Andes, Ecuador, sexual dimorphism

\section{Resumen}

Describimos la hembra de Andinosaura kiziriani de una localidad topotípica, en el sector de "El Chorro" en el suroccidente de la provincia del Azuay, Ecuador. La hembra fue encontrada entre la hojarasca y rocas, junto a la caída de agua que le da nombre al sector. El individuo se diferencia de los machos por presentar 31 filas transversales de escamas dorsales, ausencia de poros femorales, una longitud rostro-cloacal de 66,1 mm y, un patrón de coloración dorsal marrón oscuro con una banda dorsolateral, y vientre marrón oscuro con franjas o manchas blancas dispuestas longitudinalmente. Así, nuestro estudio evidencia el dimorfismo sexual en esta especie y amplía el conocimiento de sus características morfológicas y ecológicas.

Palabras clave: Lagartijas microteidas, Andes, Ecuador, dimorfismo sexual 


\section{INTRODUCCIÓN}

Los recientes estudios en lagartijas, principalmente en la familia Gymnophthalmidae en las estribaciones orientales y occidentales de América del Sur, han dado como resultado un incremento en el conocimiento de la diversidad y relaciones evolutivas entre los diversos géneros y especies de este grupo [1-7]. El género Andinosaura [6] está representado por 11 especies, distribuidas sobre los 1000 metros de elevación, exclusivamente en los Andes de Colombia y Ecuador [8]. Ocho especies se encuentran en Ecuador y seis de ellas son endémicas; a excepción de una especie, las especies se encuentran al occidente de la Cordillera de los Andes [9].

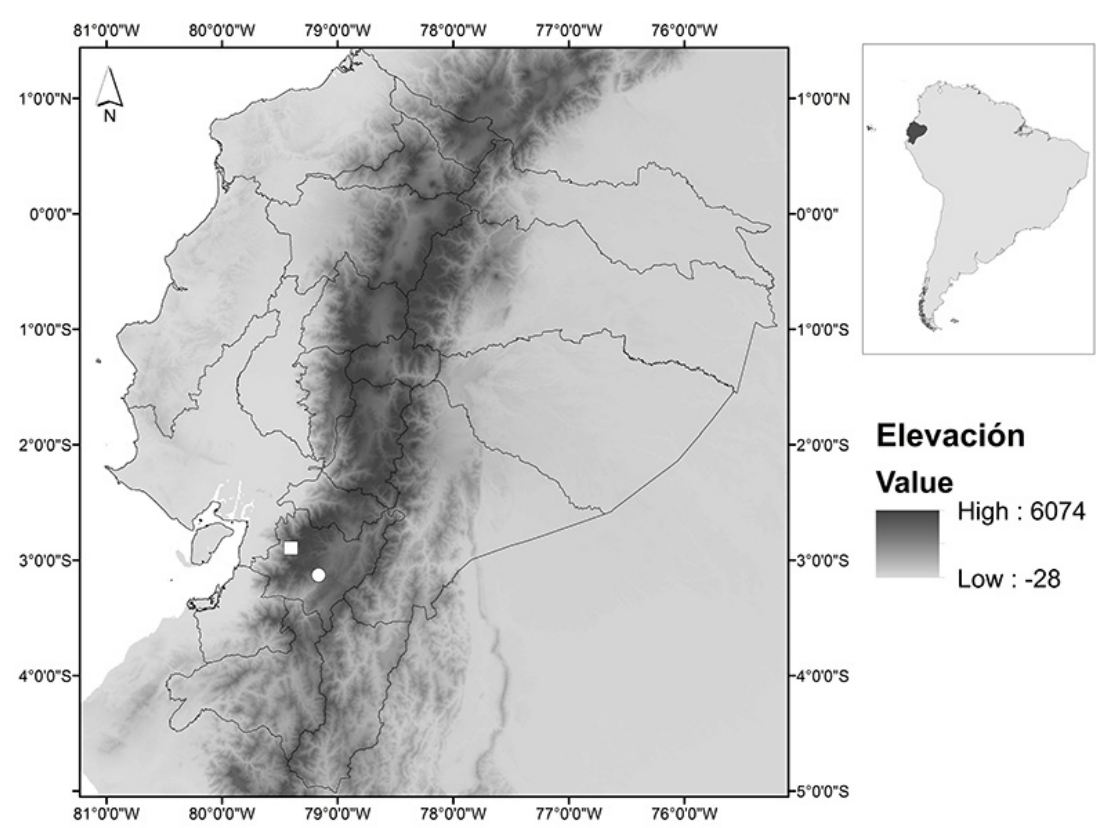

FIGURA 1. Mapa de distribución Andinosaura kiziarani en el Ecuador. Cuadrado blanco, QCAZ 9667 (holotipo) círculo blanco QCAZ 9607 (paratipo) y MZUA.RE.0287 (alotipo).

En el suroccidente del Ecuador se han registrado tres especies de Andinosaura: A. aurea, A. vespertina y A. kiziriani $[5,9]$. Sin embargo, la descripción de A. kiziriani se basó en dos individuos machos [5] y, hasta el momento, no existe información publicada formalmente de la morfología de las hembras de esta especie. Durante una visita de exploración a la localidad topotípica de A. kiziriani se encontró una hembra perteneciente a esta especie. En esta publicación contribuimos con la descripción de esta hembra, aportando información acerca de las características morfológicas y dimorfismo sexual con el fin de contribuir en la comprensión de estas especies poco conocidas. 


\section{MATERIALES Y MÉTODOS}

El individuo fue colectado en la localidad de "El Chorro de Girón" (37'46.15"S, 79 9'55.49"O, 2592 m de elevación), cantón Girón, provincia del Azuay (Fig. 1), bajo el permiso de investigación N¹17-2016-DPAA/MA] otorgado por el Ministerio del Ambiente de Ecuador y se encuentra depositado en el Museo de Zoología de la Universidad del Azuay (MZUA), Cuenca, Ecuador. El sistema de clasificación de ecosistemas utilizado para caracterizar el hábitat de la especie sigue la propuesta del Ministerio del Ambiente de Ecuador (MAE) [10]. Las localidades, coordenadas y elevaciones fueron tomadas con un GPS Garmin Etrex 10. Las fotografías fueron tomadas por Juan Carlos Sánchez (JCSN).

Seguimos la terminología y definición de presencia/ausencia y/o conteos o mediciones propuestas por Kizirian [7]: Longitud frontonasal-frontal, sutura nasoloreal, supraoculares, superciliares, postoculares, postparietales, temporales supratimpánicas, labiales, geneiales, hileras de escamas dorsales, filas longitudinales de escamas dorsales, filas transversales de escamas dorsales, filas transversales de escamas ventrales, filas longitudinales de escamas ventrales, hileras de escamas laterales, poros femorales, escamas entre los poros femorales, subdigitales, y escamas de la placa cloacal. La identificación de la especie y clasificación sistemática siguen a Sánchez-Pacheco et al. $[5,6]$. Las medidas morfológicas fueron tomadas con un calibrador digital (precisión 0,1 $\mathrm{mm}$ ) y siguen las abreviaciones longitud rostro-cloaca [LRC] y longitud de la cola [LC]. La denominación de "Alotipo" es asignado al ejemplar por ser del sexo opuesto al holotipo y no tiene condición de portanombre [11].

El ejemplar fue sacrificado con una solución de roxicaína al 10\%, fijado en una solución de formaldehído al 10\% y preservado en alcohol etílico al 75\%. La determinación del sexo se basó en la inspección directa de órganos sexuales mediante una incisión en la base de la cola. La identificación y el conteo de escamas se realizaron a través del uso de un estéreo microscopio. Los patrones de coloración en vida y demás características importantes fueron tomados de las notas de campo y fotografías. 


\section{RESULTADOS}

Andinosaura kiziriani (Sánchez-Pacheco et al., 2012) (Fig. 2-4)

Riama kiziriani Sánchez-Pacheco, 2012.
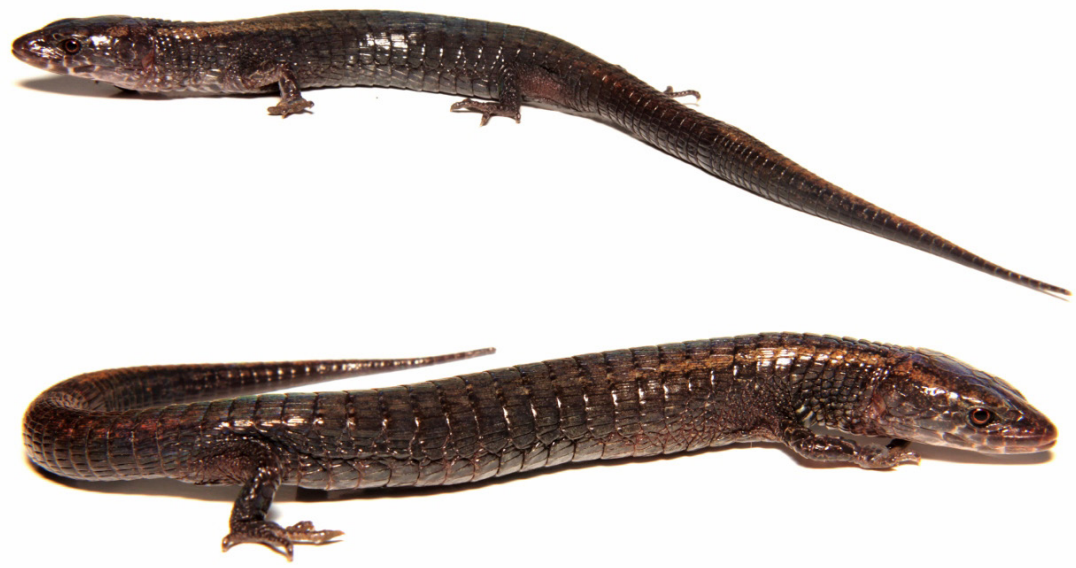

FIGURA 2. Alotipo de Andinosaura kiziriani hembra ( $\mathrm{LRC}=66,1 \mathrm{~mm})$

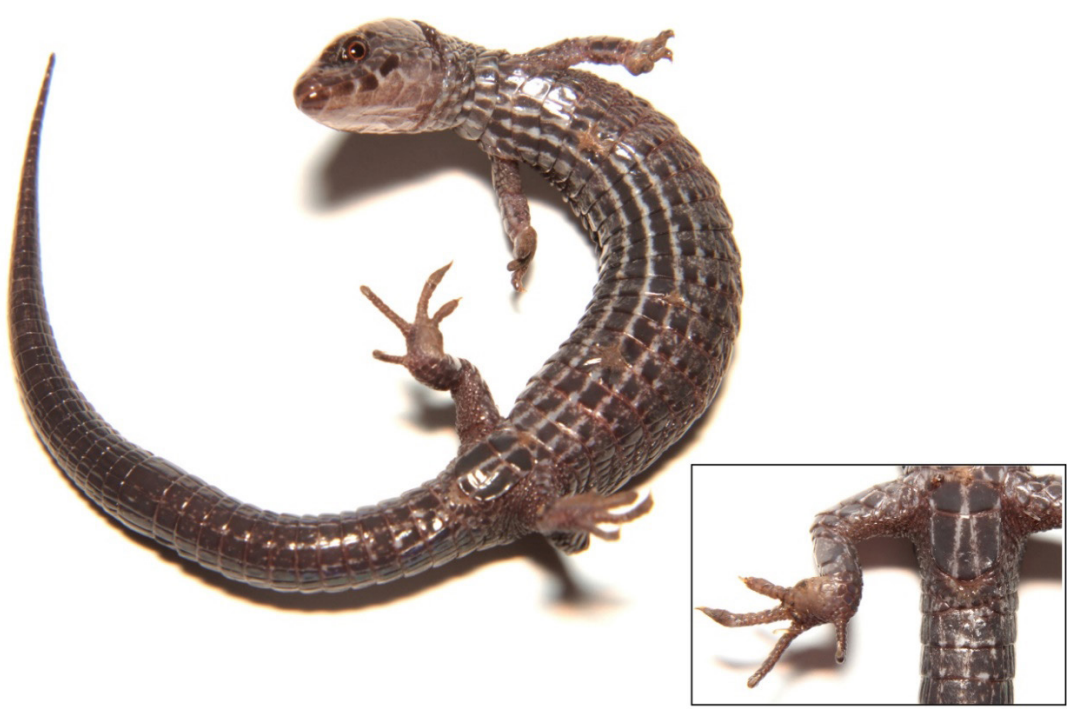

FIGURA 3. Vista ventral y detalle de la placa cloacal (recuadro inferior) del alotipo de Andinosaura kiziriani (MZUA.RE.0287). 

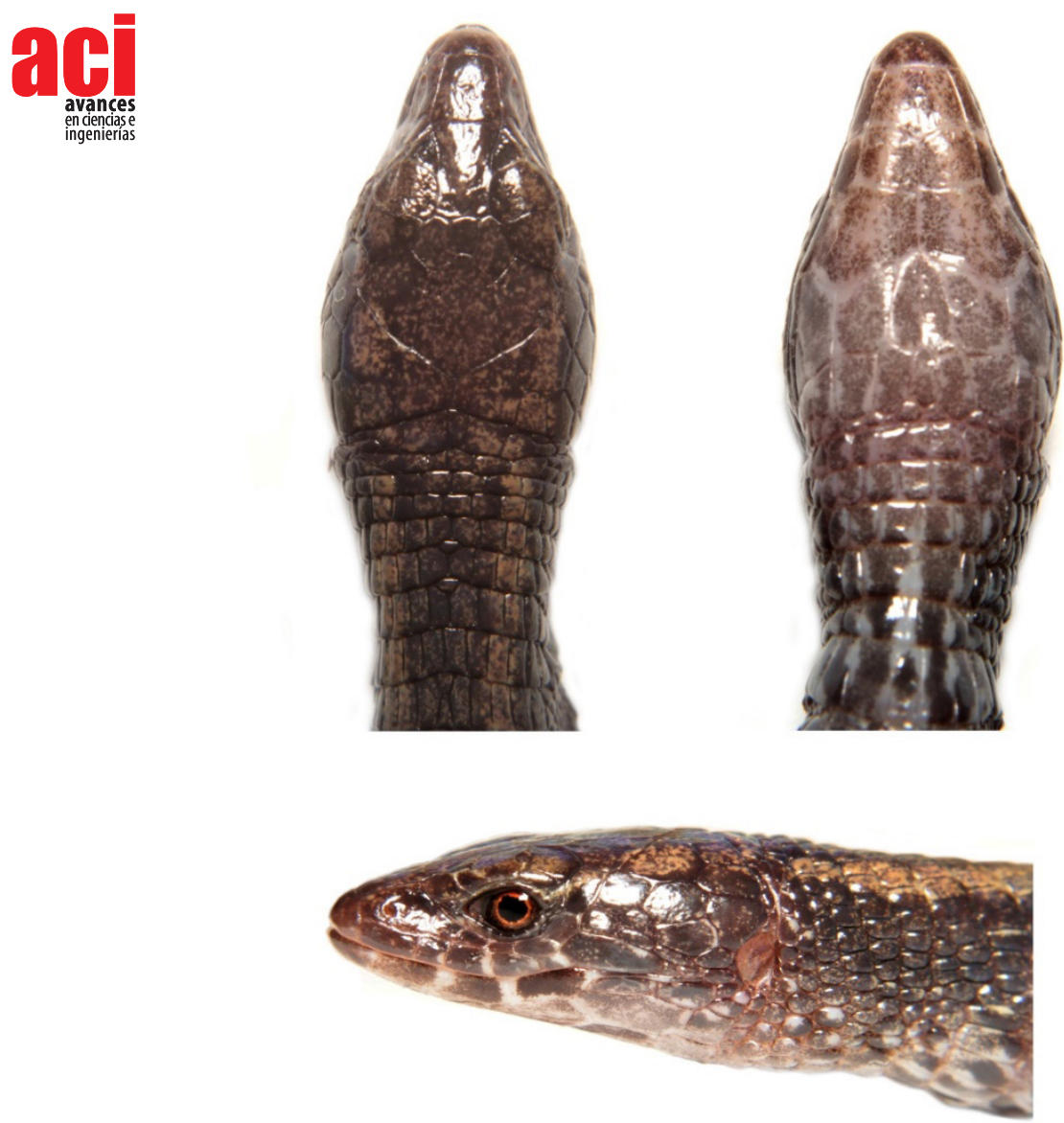

FIGURA 4. Detalle de la cabeza en vista dorsal, ventral y perfil del alotipo de Andinosaura kiziriani (MZUA.RE.0287).

Alotipo: MZUA.RE.0287 hembra adulta, colectada en el "Chorro de Girón" (37'46.15"S, $79^{\circ} 9^{\prime} 55.49^{\prime \prime} \mathrm{O}, 2592$ m de elevación), cantón Girón, provincia de Azuay, Ecuador, por José Manuel Falcón (JMF), el 01 de marzo 2016.

Diagnosis: la hembra de Andinosaura kiziriani se distingue por las siguientes características: (1) LRC 66,1 mm, LC 56,0 mm; (2) escama frontonasal menor que frontal; (3) escamas prefrontales ausentes; (4) sutura nasoloreal ausente; (5) tres escamas supraoculares, la segunda en contacto con las ciliares; (6) serie supraciliar incompleta $(=2+1)$; (7) ausencia de fusión supralabial-subocular; (8) escamas postoculares (=2); (9) escamas postparietales (=2); (10) escamas temporales supratimpanicas (=3); (11) escamas geneiales (2 pares); (12) escamas dorsales rectangulares, yuxtapuestas, estriadas; (13) escamas nucales lisas; (14) filas longitudinales de escamas dorsales (=20); (15) filas transversales de escamas dorsales (=31); (16) escamas ventrales lisas; (17) filas transversales de escamas ventrales $(=19) ;(18)$ filas de escamas laterales $(=3) ;(19)$ 
poros femorales ausentes; (20) escamas subdigitales en el dedo I del pie no visibles; (21) placa cloacal anterior con escamas pares; (22) dorso marrón oscuro, banda dorsolateral presente; vientre marrón oscuro con franjas o manchas blancas dispuestas longitudinalmente en los suturas de las escamas.

La hembra MZUA.RE.0287 de A. kiziriani (datos en paréntesis) presenta diferencias en relación a los machos en: el número de filas transversales de escamas dorsales de 32 a 34 (31); siete poros femorales en cada extremidad y seis escamas entre los poros (ausentes); LRC $(\max )=61.0 \mathrm{~mm}(\mathrm{LRC}=66.1 \mathrm{~mm})$; puntos blancos a lo largo de los flancos y cola (sin puntos blancos en flancos y cola).

Coloración en vida: (Fig.2-4). Dorso y flancos café oscuro, presenta líneas dorsolaterales café claro que cubren desde las escamas supraoculares, donde son un poco difusas, hasta casi la mitad del cuerpo donde, se van desvaneciendo hasta unificarse con la coloración dorsal de fondo. Escamas ventrales gris oscuro (casi negro) con líneas delgadas cremas grisáceas que se extienden longitudinalmente desde la garganta hasta la placa cloacal. Iris anaranjado, el borde en contacto con la pupila se presenta irregular.

Coloración en preservado: Dorsalmente la coloración es gris oscuro uniforme; en la cabeza se puede notar levemente las líneas laterales color gris claro. Ventralmente, desde la región gular hasta la cola de color gris oscuro, los márgenes de las escamas ventrales son de color gris claro, cabeza gris cenizo claro con los bordes de las escamas de color crema grisáceo.

Hábitat y ecología: La hembra de Andinosaura kiziariani MZUA.RE.0287 fue colectada en El Chorro de Girón, misma localidad de uno de los dos sitios tipo de donde se describió la especie; se la encontró aproximadamente a $100 \mathrm{~m}$ del sitio de captura del paratipo (QCAZ 9607). La zona corresponde al Bosque Siempreverde Montano de la Cordillera Occidental de los Andes. La localidad se encuentra a una elevación de 2592 metros y presenta un hábitat dominado por leñosas con abundante cobertura de briófitos y epífitos; a su vez, el terreno presenta una marcada pendiente. El espécimen se encontraba activo, dentro de un claro del bosque cercano a la orilla del río en la base de la caída de agua, donde fue capturado entre la hojarasca y rocas a las 16:00 horas aproximadamente. Durante la inspección del ejemplar en laboratorio, no se observó la presencia de huevos.

\section{DISCUSIÓN}

Las diferencias en el número y la presencia de poros femorales entre machos y hembras han sido ampliamente reportadas en lagartijas del género Andinosaura, destacando principalmente la reducción e incluso la ausencia de poros femorales $[4,5,7]$, característica que comparte la hembra de A. kiziriani. Otra característica que presenta la hembra de $A$. kiziriani es un mayor tamaño corporal que los machos, como ocurre en A. petrorum y $A$. vespertina $[5,7]$.

Las diferencias en los poros femorales han sido ampliamente utilizadas en descripciones taxonómicas [12]. Sin embargo, la presencia de poros femorales está asociada al 
comportamiento y señales químicas en saurios $[13,14]$. En este contexto, las diferencias que hemos descrito en la hembra de A. kiziriani, además de las diferencias en tamaño y patrones de coloración, aportan a la comprensión general e indicios de diferencias en la ecología de esta poco conocida especie y del grupo en general.

A pesar de ser de una localidad topotípica, el escaso número de registros conocidos de A. kiziriani (3, incluyendo el de este estudio) indican el poco conocimiento sobre la ecología y distribución de este taxón, por lo adicional.

\section{AGRADECIMIENTOS}

Este registro se realizó como parte del programa de investigación Anfibios y Reptiles del Sur de Ecuador. JMF agradece a Alejandra Recalde por su ayuda durante la fase de campo. JCSN agradece particularmente a Omar Torres-Carvajal y Santiago Sánchez-Pacheco por su ayuda en la identificación de la especie y sus comentarios. Los autores agradecen al Museo de Zoología de la Universidad del Azuay por el acceso a sus colecciones. Este trabajo fue revisado por Carolina Reyes-Puig y Juan M. Guayasamin.

\section{CONTRIBUCIONES DE LOS AUTORES}

José Manuel Falcón: diseño de metodología en campo y curación de datos; Juan Carlos Sánchez: identificación y revisión del material colectado, toma de datos y análisis de datos; Juan Carlos Sánchez y José Manuel Falcón redactaron el manuscrito. 


\section{REFERENCIAS}

[1] Torres-Carvajal, 0., Lobos, S. E., Venegas, P. J., Chávez, G., Aguirre-Peñafiel, V., Zurita, D., \& Echevarría, L. Y. (2016). Phylogeny and biogeography of the most diverse clade of South American gymnophthalmid lizards (Squamata, Gymnophthalmidae, (ercosaurinae). Molecular Phylogenetics and Evolution, 99, 63-75.

[2] Goicoechea, N., Padial, J. M., Chaparro, J. C., Castroviejo-Fisher, S., \& De la Riva, I. (2012). Molecular phylogenetics, species diversity, and biogeography of the Andean lizards of the genus Proctoporus (Squamata: Gymnophthalmidae). Molecular Phylogenetics and Evolution, 65(3), 953-964.

[3] Torres-Carvajal, 0., \& Mafla-Endara, P. (2013). Evolutionary history of Andean Pholidobolus and Macropholidus (Squamata: Gymnophthalmidae) lizards. Molecular Phylogenetics and Evolution, 68(2), 212-217.

[4] Sánchez-Pacheco, S. J., Kizirian, D. A., \& Nunes, P. M. S. (2011). A new species of Riama from Ecuador previously referred to as Riama hyposticta (Boulenger, 1902)(Squamata: Gymnophthalmidae). American Museum Novitates, (3719), 1-15.

[5] Sánchez-Pacheco, S. J., Aguirre-Peñafiel, V., \& Torres-Carvajal, 0. (2012). Lizards of the genus Riama (Squamata: Gymnophthalmidae): The diversity in southern Ecuador revisited. South American Journal of Herpetology, 7(3), 259275.

[6] Sánchez Pacheco, S. J., Torres Carvajal, 0., Aguirre Peñafiel, V., Nunes, P. M., Verrastro, L., Rivas, G. A., Rodriguez, M. T., Grant, T., \& Murphy, R. W. (2017). Phylogeny of Riama (Squamata: Gymnophthalmidae), impact of phenotypic evidence on molecular datasets, and the origin of the Sierra Nevada de Santa Marta endemic fauna. Cladistics, 34(3), 260-291.

[7] Kizirian, D. A. (1996). A review of Ecuadorian Proctoporus (Squamata: Gymnophthalmidae) with descriptions of nine new species. Herpetological monographs, 85-155.

[8] Uetz, P., Hošek, J., \& Hallermann, J. (2016). The reptile database. Recuperado de: http://www.reptile-database.org/ el $27 / 02 / 2020$

[9] Torres-Carvajal, 0., Pazmiño-Otamendi, G. y Salazar-Valenzuela, D. 2020. Reptiles del Ecuador. Versión 2020. Museo de Zoología, Pontificia Universidad Católica del Ecuador. Recuperado de: https://bioweb.bio/faunaweb/reptiliaweb el $27 / 02 / 2020$

[10] Ministerio del Ambiente del Ecuador (2012). Sistema de clasificación de los ecosistemas del Ecuador continental. Subsecretaría de Patrimonio Natural. Quito.

[11] Zoológica, N. (1999). Código Internacional de Nomenclatura Zoológica.

[12] Baeckens, S., Edwards, S., Huyghe, K., \& Van Damme, R. (2015). Chemical signalling in lizards: an interspecific comparison of femoral pore numbers in Lacertidae. Biological journal of the Linnean Society, 114(1), 44-57.

[13] Mason, R. C. (1992). Biology of the Reptilia, Vol. 18, physiology E hormones, brain, and behavior. Chicago: University of Chicago Press

[14] Van Wyk, J. H., \& le FN Mouton, P. (1992). Glandular epidermal structures in cordylid lizards. Amphibia-Reptilia, 13(1), 1-12. 